\title{
Frontiers in Respiratory Physiology - grand challenge
}

\section{Paul W. Davenport ${ }^{1}$, Andreas von Leupoldt ${ }^{2}$, Karen Wheeler-Hegland ${ }^{1}$ and lan M. Colrain ${ }^{3}$}

\author{
1 University of Florida, Gainesville, FL, USA \\ 2 University of Hamburg, Hamburg, Germany \\ ${ }^{3}$ SRI International, Menlo Park, CA, USA \\ *Correspondence: pdavenpo@ufl.edu
}

Respiration is one of the most integrated and fundamental bodily processes in humans and animals. The respiratory system provides the organism with oxygen necessary for all vital functioning and removes metabolic byproducts such as carbon dioxide. Failure of the respiratory system is related to malfunction of many organ systems with severe consequences throughout the organism. Over the past decades, respiratory physiology research has greatly improved our understanding of the respiratory system by using both human and animal models of respiratory function and disease. Reductionist methodologies have provided increasing understanding of mechanisms of respiratory tissue, mechanics, gas exchange, and neural control. Advances in molecular and genomic research have led to broadening our understanding of the cellular mechanisms of the respiratory system and body homeostasis. Constructionist methodologies have integrated diverse mechanistic aspects of respiratory function into a better understanding of integrated respiratory behaviors. However, previous findings were often restricted to one specific research discipline and poorly integrated with findings from other research specialties, although the overarching theme remains the respiratory system. The dynamic tension between reductionist and constructionist research produces an iterative pathway to a more complete understanding of respiratory physiology. The challenge facing us in the 21 st century is to build on major advances by applying new reductionist technologies and integrating that information in constructionist development of whole body respiratory function. The mission of this journal is to bring together the divergent disciplines and methodologies examining respiratory physiology in a forum that will lead to an integrated understanding of the respiratory system.

Respiratory physiology researchers have used multiple new or well established methodologies to address the critical features of our research hypotheses. Hypothesis testing is the basic foundation of all our research. Methodologies were developed and will be developed as physiologists build our body of knowledge and understanding of respiratory physiology. Hypothesis testing is the foundation of all science that moves our field forward and is fundamental to research presented in this journal. There are multiple ways to approach new research questions and there are multiple systems which must be investigated to better understand respiratory physiology. The challenge before us is to embrace existing technologies and invent new technologies while keeping the hypothesis at the center of our research.

Another challenge we face is increasing complexity of our mechanistic understanding of the respiratory system and its integration with other body systems. We know that the respiratory system is more than just lungs and airways, it includes the inspiratory pump, the neural systems that control the movement and distribution of gases from the lung into the cardiovascular system, supply and removal of the blood gases essential for life and the psychological and behavioral consequences of normal and disordered breathing. A unique aspect is the respiratory system constantly having to meet homeostatic drives while also being under volitional and unconscious control to serve important behavioral functions. Speech, singing, breath-holding, regulation of breathing for athletic endeavors all require involvement of cortical and subcortical areas of the central nervous system outside those responsible for ventilation and basic homeostatic regulation. Likewise, sensation and perception of respiratory signals is enhanced relative to that experienced in relation to other basic physiological functions, again highlighting a cortical role in the processing of respiratory afferent information. Therefore, it is clearly important to understand that the respiratory system functions as an integral component of all other physiological systems within the body.
A major challenge for future research is to continue the discovery of those mechanisms and processes which integrate respiratory function with the whole organism.

With the increasing complexity of our understanding respiratory physiology we recognize that multiple areas must be embraced for our field to grow. This journal has a mission to include at least several major areas of emphasis of our research as a forum for publishing advances in our knowledge of respiratory physiology. Systemic respiratory physiology needs to engage the behavioral control of breathing and the neural basis for psychophysiology, respiratory pattern, sensations and reflexes. Central airway protection (CAP) is a fundamental process for preventing or ejecting material that could penetrate the lung producing pathologies. Research is needed to provide a better understanding of individual CAP reflexes and their control of respiration such as swallow, laryngeal adduction, expiratory reflex, and cough. This must be further extended into the integration and coordination of CAP mechanisms with neural control and behavioral control of breathing. Respiration does not occur without pumping, generated by the respiratory muscles. New research needs to be reported identifying muscle control mechanisms, muscle rehabilitation, muscle degradation in disease, and muscle molecular mechanisms. The mechanical changes generated by the respiratory muscle pumping forces interact with lung and tissue mechanics producing the pressure driving forces for air to move in and out of the lung. Much is known about respiratory mechanics yet much more needs to be discovered and reported. Lung and airway biological mechanisms which modulate respiratory mechanics, shape the pressure-flow-volume characteristics of air movement and alterations with lung pathology must continue to be investigated. Ultimately, molecular concentrations of oxygen and carbon dioxide within the alveolar space set the driving forces for gas exchange with the blood. 
The interface for gas exchange and oxygen/ carbon dioxide transport is fundamental to delivering oxygen to the tissues and removal of metabolic byproducts. These mechanisms develop throughout an animal's life history from fetus to old age. New information on the life-history progression of the respiratory system is needed for us to expand our understanding of life-stage dependent respiratory function. Our research must use multiple models from non-invasive human studies to invasive animal studies to mathematical modeling of the respiratory system. Continued development of models of the respiratory system will allow us to engage new techniques for investigating the mechanisms predicted by these models. Adequate modeling of respiratory components will also predict methods of interaction using pharmacological agents and environmental impacts on respiratory function. All models have both functional and structural components, hence, essential research must be reported on the structure and ultrastructure of the respiratory system and the relationship between structure and function. Finally, when the system fails or a component of the system fails, the ability of the body to survive is compromised. The relationship between pathophysiology and respiratory physiology can provide new insights into fundamental function of the respiratory system as an integrated component of body homeostasis. This Journal has the challenge of providing a forum for reporting new knowledge in these divergent and convergent respiratory physiology disciplines.

We are now in the new environment of dissemination of our research results to our colleagues. Reporting of our research is rapidly moving from paper libraries to digital libraries. The availability of research results with new online, open-access journals such as the Frontiers in Respiratory Physiology engages researchers worldwide with immediacy unforeseen a few decades ago. However, we should not ignore our research roots, housed in journals in our paper libraries. We are convinced that although it will remain our responsibility to remember and utilize our research foundations published in traditional paper libraries - the present new online, open-access model of publishing new discoveries will greatly contribute to increase our knowledge on respiratory physiology.

Received: 16September 2010; accepted: 17 September 2010; published online: 25 October 2010.

Citation: Davenport PW, von Leupoldt A, WheelerHegland Kand Colrain IM (2010) Frontiers in Respiratory Physiology - grand challenge. Front. Physio. 1:139. doi: 10.3389/fphys.2010.00139

This article was submitted to Frontiers in Respiratory Physiology, a specialty of Frontiers in Physiology.

Copyright () 2010 Davenport, von Leupoldt, WheelerHegland and Colrain. This is an open-access article subject to an exclusive license agreement between the authors and the Frontiers Research Foundation, which permits unrestricted use, distribution, and reproduction in any medium, provided the original authors and source are credited. 